\title{
RECONSTRUCTION OF NASAL ALA DEFECTS USING THREE STAGED FOLDED FOREHEAD FLAP: AESTHETIC AND FUNCTIONAL OUTCOMES
}

\author{
Ghazanfar Ali, Moizza Tahir, Maqbool Raza, Danish Almas*, Abdul Majid**, Yasser Saeed Khan***
}

Combined Military Hospital Multan/National University of Medical Sciences (NUMS) Pakistan, *Combined Military Hospital Bahawalpur/ National University of Medical Sciences (NUMS) Pakistan, ${ }^{* *}$ Combined Military Hospital /National University of Medical Sciences (NUMS) Rawalpindi Pakistan, ${ }^{* * *}$ Combined Military Hospital Quetta/National University of Medical Sciences (NUMS) Pakistan

\section{ABSTRACT}

Objective: To assess short and long term aesthetic and functional outcomes of nasal ala reconstruction by three staged folded forehead flap.

Study Design: Prospective study.

Place and Duration of Study: Plastic and Reconstructive Surgery Department of Combined Military Hospital Bahawalpur and Quetta, from Dec 2014 to Dec 2018.

Methodology: Total of 29 patients were enrolled through consecutive sampling technique. Nasal ala defects were reconstructed by three staged frontal forehead flap under general anesthesia. Follow up visit at 2 weeks bleeding, wound Infection, partial skin graft loss and partial flap necrosis was documented. Follow up at 24 weeks, functional outcome of nasal reconstruction was assessed by history and clinical examination for nasal obstruction and/or crusting. Patients' subjective satisfaction about aesthetic outcome of nasal reconstruction was documented on 5 point likert scale. Objective aesthetic assessment of reconstruction was done on 2D photograph by two independent surgeons.

Results: Twenty six (89.66\%) males and 3 (10.34\%) females participated in study. The mean age was 35 years range 16 to 64 years. Follow up at two weeks revealed postoperative bleeding in $2(6.8 \%)$, wound infection in 1 $(3.4 \%)$ and partial flap loss in $1(3.4 \%)$ patient. At 24 weeks follow up all patients had patent nasal ala. Subjective and objective assessment of nose shape was documented.

Conclusion: Use of three staged folded forehead flapis useful method of reconstruction for full thickness defects of nasal alaewith good functional and aesthetic outcome.

Keywords: Aesthetic outcomes, Cartilage, Forehead flap, Nasal ala reconstruction.

This is an Open Access article distributed under the terms of the Creative Commons Attribution License (http://creativecommons.org/licenses/by/4.0), which permits unrestricted use, distribution, and reproduction in any medium, provided the original work is properly cited.

\section{INTRODUCTION}

Face looks attractive when every feature of the face is symmetrically placed. Nose is the primary feature of the face. It forms central projection of face. Anatomically nose is acomposite structure having skeletal and soft tissue components. The nasal surface is not uniform. It is divided into aesthetic subunits-the dorsum, tip, columella, and the paired sidewalls, alae, and soft triangles-based on characteristic skin quality, border outline, and three-dimensional contour ${ }^{1}$. Nasal ala skin is thin and adherent. It contains no cartilage, but for restoration of its anatomy, insertion of cartilage gives better aesthetic appeal. There are multiple etiologies of nasal ala defects.

Correspondence: Dr Ghazanfar Ali, Plastic Surgeon, Combined Military Hospital, Multan Pakistan

Received: 04 Apr 2020; revised received: 10 Aug 2020; accepted: 17 Aug 2020
Excision of skin cancer, especially basal cell carcinoma is the commonest etiology of nasal ala defects 2 followed by trauma, infections, and congenital defects.

Reconstruction of nose is challenging not only because of its aesthetic position, but also to maintain its function. The nasal repair and reconstruction demand careful analysis of anatomical, aesthetic and cultural aspects. Staged surgeries may be required. Initial surgery may be done to achieve clear margins by routine surgery or Mohs surgery, recreate the defect, reconstruct nasal platform and prepare tissues for transfer. Large nasal defects of ala reconstruction is carried in three layers; skin, inner lining and cartilage. Various options for reconstruction of nasal ala are healing with secondary intention and free tissue transfer. Skin cover can be provided by folded 
forehead, naso-labial or free radial forearm flap. The skeletal support can be provided by conchal cartilage, nasal bone or rib graft ${ }^{2}$. Nasal lining can be provided by multiple options including; intranasal flaps, skin grafts, downturned flaps prelaminated forehead flaps, folded forehead or naso-labial flaps and free flaps 3,4 .

Composite nasal ala defects pose unique challenge; it has no skeletal structure in it but reconstruction requires skeletal support for proper functional and aesthetic aspects. The versatile concha cartilage is first choice for nasal ala reconstruction. It is thin, pliable and donor site morbidity is minimal. Moreover, the donor area scar is almost inconspicuous. Three staged folded forehead flap can be used for provision of skin coverage and lining. It has best color and texture match to the lost nasal skin. There is minimal donor site morbidity as wound can be closed primarily in most of the cases.

To date, thereare limited publications that have assessed the outcomes of the three-stage folded forehead flap (FFF) for lining reconstruction with regard to thenose shape and function ${ }^{3}$. The objective of this study is to share experience of three-staged FFF for nasal reconstruction and assess short and long term aesthetic and functional outcomes of nasal ala reconstruction by three staged folded forehead flap. The present study would improve practice.

\section{METHODOLOGY}

This study was conducted at the Plastic and Reconstructive Surgery Department of Combined Military Hospital Bahawalpur and Quetta from December 2014 to December 2018 after hospital ethical committee's approval. Total of 29 patients were enrolled in study through consecutive sampling technique. Patients with full thickness unilateral nasal ala defects were included in the study. Patients with chronic renal failure, heart disease and smoking were excluded.

Pre-operatively patients were counseled about staged surgery under general anesthesia, hospitalstay and follow up visit inoutpatient department (OPD). Patients were informed about possible complications of bleeding, partial or total flap loss, revision surgery, loss of sensations around forehead, open wound and scar on forehead. The defects were analyzed and reconstruction was performed in three stages using FFF and conchal cartilage.

Post-surgical followedup was done at 2 week (early) and at 24 weeks (Late). At 2 weeks follow up assessment for bleeding, wound infection, partial skin graft loss and partial flap necrosis was done. At 24 weeks nose shape was assessed by $2 \mathrm{D}$ photograph in a standardized setting. Aesthetic outcome was assessed by reconstructed patients individually as well as by 2 surgeons from other departments who were blinded to the purpose of the study. Patients' subjective aesthetic satisfaction was measured using a 5-point likert scale $(5=$ significantly satisfied, $1=$ dissatisfied). Functional outcome of nasal reconstruction was assessed by history and clinical examination for nasal obstruction and/or crusting. The data was interpreted and analyzed by using SSPS-20.

\section{Surgical Technique}

After excision of the tumor or debridement of nasal trauma wound, defects were analyzed and templates were made accordingly as a part of reverse planning. The forehead flaps were marked according to the defects. Supraorbital and supratrochlear arteries were marked with the help of hand held Doppler ultrasound. One percent lignocaine with adrenaline local anesthetic agent was injected for better hemostasis before flap elevation. The forehead flap was raised in supra-periosteal plane. However periosteum was included with the flap staring from $1 \mathrm{~cm}$ above the supra-orbital rim. The hemostasis was secured meticulously and the FFF was inset using vicryl 5/0 for intra-nasal suturing and proline $5 / 0$ for skin closure. The raw surface is covered with partial thickness skin graft. The donor site was closed primarily in most of the cases after undermining of skin.

The second stage is carried out after 6 weeks. During second stage the flap was raised along the alar rim and thinning was carried out. This was 
followed by insertion of conchal cartilage. A template of the nasal ala was made and conchal cartilage was harvested, $1 \%$ lignocaine with adrenaline was used for hemostasis. The third stage was performed 6 weeks later. Flap was inset after division of the pedicle. At the base of the forehead flap wound was closed to balance eyebrows and achieve minimal scar at donor site.

\section{RESULTS}

Total of 29 patients, $26(89.66 \%)$ males, and 3 $(10.34 \%)$ females participated in study. The mean age was 35 years (range 16 to 64 ) years. The

Table: Follow up results at 2 weeks.

\begin{tabular}{l|c}
\hline Complication & $\mathbf{n ~ ( \% )}$ \\
\hline Bleeding & $2(6.9 \%)$ \\
\hline Wound Infection & $1(3.45 \%)$ \\
\hline Partial Flap Necrosis & $1(3.45 \%)$ \\
\hline Total Flap Necrosis & - \\
\hline
\end{tabular}

results of early follow up at 2 weeks are listed in table. Follow up at 24 weeks subjective aesthetic satisfaction measured through a 5-point satisfaction scale revealed that 22 patients $(75.8 \%)$ aesthetic outcome revealed an excellent result in 7 patients $(24.1 \%)$, a good result in 15 patients $(51.7 \%)$ a fair result in 5 patients $(17.2 \%)$, and a poor result in 2 patients (12\%). Functional assessment of nose revealed no nasal obstruction/or crusting at 24 weeks follow up history and clinical examination.

\section{DISCUSSION}

In nasal reconstruction aesthetic and cultural aspects are considered for an elegant solution. Closure options are individualized for each patient and defect. Paramedian forehead flap of nasal reconstruction is not a novice technique. First described in $700 \mathrm{BC}$ in ancient Indian literature by Sushruta Samita to reconstruct nasal defects from punitive tip amputation, it has subsequently become a workhorse of nasal reconstruction requiring more than $2 \mathrm{~cm}$ soft tissue replacement on the external and internal nose 5 . The subunit principle of nasal reconstruction was done in our study. This concept was popularized by Millard6, Gonzalez-Ulloa7, Burget and Menick ${ }^{8}$. Most surg-
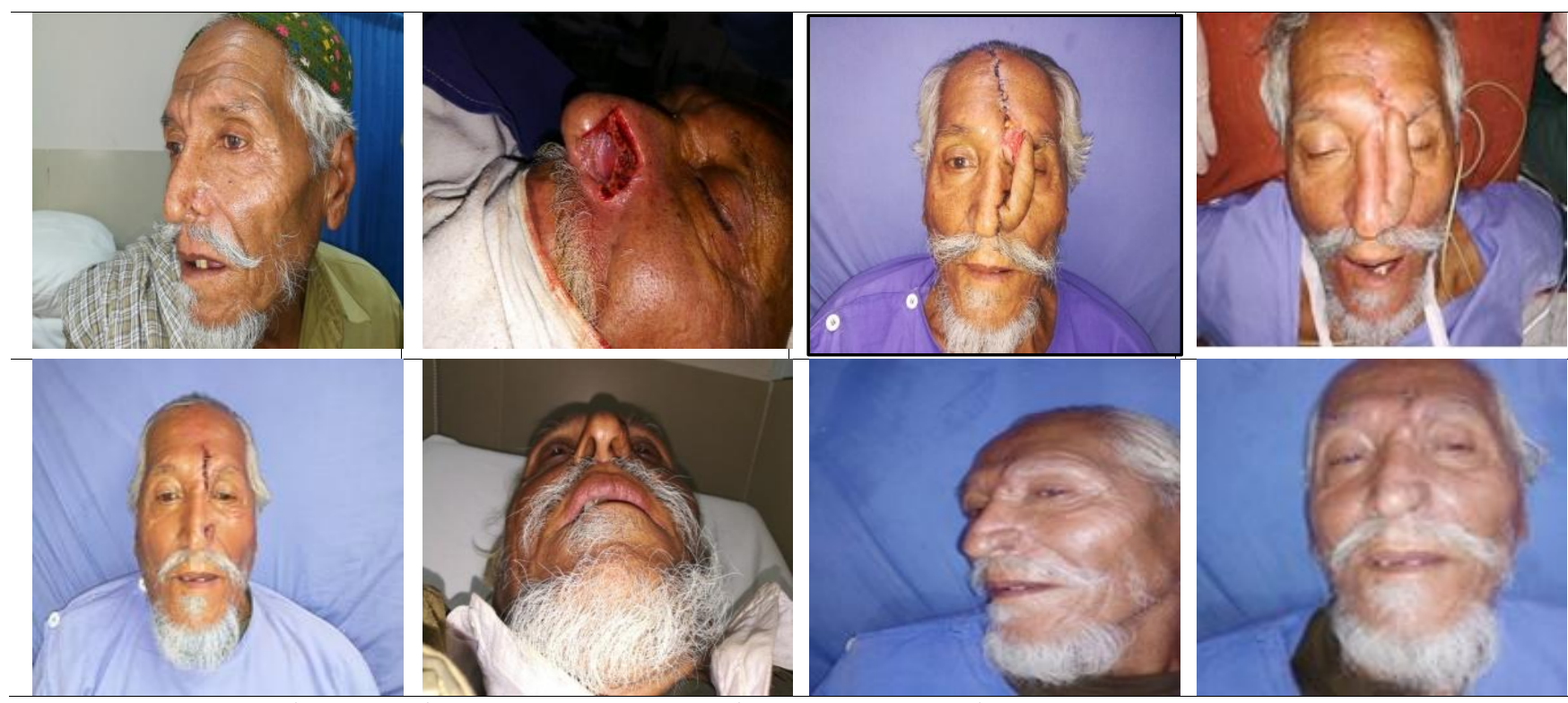

Figure: Follow up (24 weeks): The top row shows (from left to right) pre-operative, per-operative and early postoperative pictures. The bottom row shows (from left to right) pictures after division of pedicle and late follow up.

were significantly satisfied, $4(13.7 \%)$ satisfied, 1 (3.4\%) was fairly satisfied, 1 (3.4\%) equivocal and 1 patient (3.4\%) was dissatisfied. The objective eons are of the opinion that that by excising $50 \%$ or less of an aesthetic subunit, the surgeon sacrifice healthy tissue, necessitating a larger flap and 
complicating the reconstruction. But the resultant scar in the middle of subunit weakens their argument. Nasal subunits principal is vital for nasal reconstruction, but for better cosmesis skin texture, color, contour, and actinic damage, are also considered. Modification of the subunit principle may be done while focusing on above factors. This may provide a pleasantoutcome at defect and at the donor site 9 .

Bleeding from edge of the flap is the most common complication in early follow up. It can be minimized by meticulous surgical technique and coverage of the raw surface with split thickness skin graft. Wound infection was observed in $1(3.45 \%)$ case and partial loss of flap in $1(3.45 \%)$ case in our study. Boyd et al, documented partial flap necrosis in $1 \%$ of their cases and insignificant bleeding in FFP for nasal reconstruction ${ }^{10}$. Little et al, described potential complications of the PFF as bleeding, pain, poor scarring, infection, dehiscence, distortion of free margins and flap necro$\operatorname{sis}^{11}$.

Staged reconstruction of nose was performed in our study with a delay of six weeks, however a delay of 7-10 days have been documented for optimum results ${ }^{12}$. Revision and refinement of flap at 3 weeks followed by sections of pedicle at six weeks has been performed ${ }^{13,14}$.

Asian anatomy and wound healing differ from Western experiences. Hsiao et al15, used modified folded forehead flap to improve functional and aesthetic outcome in Asian population. Techniques of extension of subunit and flap boundaries, minimizing flap thinning, and overbuilding of nasal framework can overcome contraction and suboptimal scarring 15 .

Nobel et al, compared nasal reconstruction using folded forehead flap and standard forehead flap along with other method of lining found reconstructed ala thicker than the normal side. Reconstructed nostril was smaller than the normal nostril in both groups. In their study $84 \%$ patients were satisfied with functional outcome and $100 \%$ were satisfied with their nasal appearance ${ }^{3}$.
Use of forehead flap leaves a scar at the donor site. This scar may be inconspicuous with good surgical technique. Schafer et al, performed folded transposition flap from cheek instead of forehead flap. The functional outcome was similar to forehead flap but the donor scar was less obvious as compared to forehead with better aesthetic outcomes ${ }^{16}$. Aesthetic results of 3-stage forehead flap technique; in terms of flap thickness compared with the 2-stage technique was not found superior. The 2-stage technique was considered state-of-the-art choice for nasal reconstruction even in complex nasal defects ${ }^{17}$.

In our study patient satisfaction was very high. However, $3 \%$ of patients were not satisfied with the procedure. These results are not very different to the previously conducted studies. Bashir et al studied ${ }^{2}$, patients, and used forehead flap for nasal reconstruction. They reported 12 $(66.67 \%)$ patients as satisfied and $2(11.11 \%)$ patients as unsatisfied regarding airway patency. For alar rim contour only $3(16.66 \%)$ patients were satisfied while 6 (33.33\%) were unsatisfied. Stahl et $a l$, in a prospective study of 9 years documented nosuperior aesthetic outcomes in comparison of 3-stage forehead flap technique, especially in relation to flap thickness compared with the 2stage technique. They considered 2-stage technique as better choice for nasal reconstruction, even in complex defects ${ }^{17}$.

Optimal cosmetic and functional results while minimizing stages and resection of healthy tissue can be achieved. Rohrich et al, in retrospective study on nasal reconstruction described six core principles as (1) maximum tissue conservation; (2) reconstruction of the defect, not the subunit; (3) complementary procedures, such as primary dermabrasion; (4) primary defatting to decrease the number of revisionary procedures; (5) the use of axial pattern flaps; and (6) good contour is the aesthetic endpoint ${ }^{18}$.

\section{CONCLUSION}

Three staged FFF along with conchal cartilage is a reliable, versatile, and useful method of 


\section{reconstruction for full thickness defects of nasal alaewith good functional and aesthetic outcome. \\ CONFLICT OF INTEREST}

This study has no conflict of interest to be declared by any author.

\section{REFERENCES}

1. Burget GC. Nasal support and lining the marriage of beauty and blood supply. Plast Reconstr Surg 1989; 84(2): 189-02.

2. Bashir MM, Khan BA, Abbas M, Khan FA. Outcome of modified turn in flaps for the lining with primary cartilage support in nasal reconstruction. J Craniofac Surg 2013; 24(2): 454-57.

3. Noel W, Duron J, Jabbour S, Revol M, Mazouz-dorval S. Threestage folded forehead flap for nasal reconstruction: objective and subjective measurements of aesthetic and functional out-comes. J Plast Reconstr Aesthetic Surg 2018; 71(4): 548-56.

4. Burget GC, Murrel GL TD. Aesthetic reconstruction of the confluence of the nose, lip and cheek. Oper Tech Plast Reconstr Surg 1998; 5(1): 76-88.

5. Burget WR. Optimal use of mirovascular free flaps, cartilage grafts, and a paramedian forehead flap foe aesthetic reconstruction. Plast Reconstr Surg 2007; 120(5): 1171-07.

6. Millard J. Aesthetic reconstructive rhinoplasty. Clin Plast Surg 1981; 8(2): 169-75.

7. González-Ulloa M. Regional aesthetic units of the face. Plast Reconstr Surg 1987; 79(3): 489-90.
8. Burget $M$. The subunit principle in nasal reconstruction. Plast Reconst surg 1985; 76(2): 239-47.

9. Singh DJ, Bartlett SP. Aesthetic considerations in nasal reconstruction and the role of modified nasal subunits. Plast Reconstr Surg 2003; 111(2): 639-48.

10. Boyd CM, Baker SR, Fader DJ, Wang JT. The forehead flap for nasal reconstruction. Arch Dermatol 2000; 136(11): 1365-70.

11. Little SC, Hughley PS. Complications with forehead flaps in nasal reconstruction. Laryn 2009; 119(6): 1093-99.

12. Schrieber M. Elegant solutions for complex paramedian forehead flap reconstruction. Facial Plast Surg Clin North Am 2011; 19(3): 465-79.

13. Brodland DG. Paramedian forehead flap reconstruction for nasal defects. Dermatologic Surg 2005; 31(8): 1046-52.

14. Frederick J. Menick. A 10-Year experience in nasal reconstruction with the three stage forehead flap. Plast Reconstr Surg 2001; 109(6): 1839-55.

15. Hsaio YC, Chang JZ. Aesthetic refinements in forehead flap reconstruction of asian nose. Plast Surg 2017; 25(2): 71-77.

16. Schäfer K, Rudolph C, Cotofana S, Goebeler M W. Large nasal defects with exposed cartilage The Folded Flap as an Innovative Alternative to the to the Paramedian Forehead Flap. Dermatol 2018; 234(3-4): 99-04.

17. Stahl AS, Gubisch W, Haack S, Meisner C, Stahl S. Aesthetic and functional outcome of 2-stage versus 3-stage Paramedian Forehead Flap Techniques. Dermatologic Surg 2015; 41(10): 1137-48.

18. Rohrich RJ, Griffin JR, Ansari M. Nasal reconstruction beyond aesthetic subunits a 15-year review of 1334 cases. Plast Reconstr Surg 2004; 114(6): 1405-16. 